\title{
EFFECTS OF SYNTHETIC AND ENHANCED VISION TECHNOLOGIES FOR LUNAR LANDINGS
}

\author{
Lynda J. Kramer ${ }^{1}$, Robert M. Norman ${ }^{2}$, Lawrence J. Prinzel III ${ }^{1}$, Randall E. Bailey ${ }^{1}$, \\ Jarvis (Trey) J. Arthur III ${ }^{1}$, Kevin J. Shelton ${ }^{1}$, and Steven P. Williams ${ }^{1}$ \\ NASA Langley Research Center ${ }^{1}$, Hampton, VA \\ Boeing Research and Technology ${ }^{2}$, Hampton, VA
}

\begin{abstract}
Eight pilots participated as test subjects in a fixedbased simulation experiment to evaluate advanced vision display technologies such as Enhanced Vision (EV) and Synthetic Vision (SV) for providing terrain imagery on flight displays in a Lunar Lander Vehicle. Subjects were asked to fly 20 approaches to the Apollo 15 lunar landing site with four different display concepts - Baseline (symbology only with no terrain imagery), EV only (terrain imagery from Forward Looking InfraRed, or FLIR, and LIght Detection and Ranging, or LIDAR, sensors), SV only (terrain imagery from onboard database), and Fused EV and SV concepts. As expected, manual landing performance was excellent (within a meter of landing site center) and not affected by the inclusion of EV or SV terrain imagery on the Lunar Lander flight displays. Subjective ratings revealed significant situation awareness improvements with the concepts employing EV and/or SV terrain imagery compared to the Baseline condition that had no terrain imagery. In addition, display concepts employing EV imagery (compared to the SV and Baseline concepts which had none) were significantly better for pilot detection of intentional but unannounced navigation failures since this imagery provided an intuitive and obvious visual methodology to monitor the validity of the navigation solution.
\end{abstract}

\section{Introduction}

Past aviation research has demonstrated the substantial potential of synthetic vision (SV) and enhanced vision (EV) technologies to enable equivalent visual operations independent of actual weather and visibility conditions [1-4]. SV is a computer-generated image of the external scene topography, generated from vehicle attitude, high- precision navigation, and data of the terrain, obstacles, cultural features, and other required flight information presented to a pilot on his/her flight displays (e.g., head-up and head-down primary flight displays, navigation displays, and auxiliary displays such as electronic flight bags). EV is an electronic means of providing a display of the external scene by use of an imaging sensor, such as Forward-Looking InfraRed (FLIR) or LIght Detection and Ranging (LIDAR). By creating, supplementing, or enhancing the natural vision of the pilot, these vision-based technologies have been shown to significantly improve situation awareness, reduce workload, enhance aviation safety (e.g., controlled-flight-into-terrain prevention and other hazards detection), and promote flight path control precision.

The intended use of EV and SV technologies mirror each other, as they both attempt to eliminate low-visibility conditions and replicate the operational benefits of clear-day flight operations, regardless of the actual outside visibility conditions. The issues that drove the design and development of synthetic and enhanced vision have commonalities to other application domains; most notably, during descent and landing on moon and planetary surfaces.

To investigate the potential benefits of an EV and/or SV-enhanced flight deck for spacecraft, the Apollo lunar landing mission was reviewed. The Apollo astronauts were tasked with navigating a new vehicle, the Lunar Module (LM), in an unknown environment with basic flight instrumentation, mainly consisting of electro-mechanical gauges (Figure 1). Astronaut Neil Armstrong identified the landing phase as being the most difficult part of the Apollo 11 mission [5]; therefore, this experiment chose to focus on the descent to landing stage. Also, Apollo experience showed that [6] "the constraints placed on 
crew visibility by the design of the LM window and by trajectory parameters make the viewing of the programmed landing site a major problem." For the Apollo missions, pilot visibility was of the utmost importance. The lunar landing task relied on the pilot's natural vision, coupled with large forwardfacing windows and operational flight profiles which allowed the pilot to see the landing zone for extended periods of time, to perform a visual, manual landing approach or to re-designate and fly to a new landing area if the original landing site was not suitable. The Apollo LM windows provided almost 70 degrees nosedown visibility.

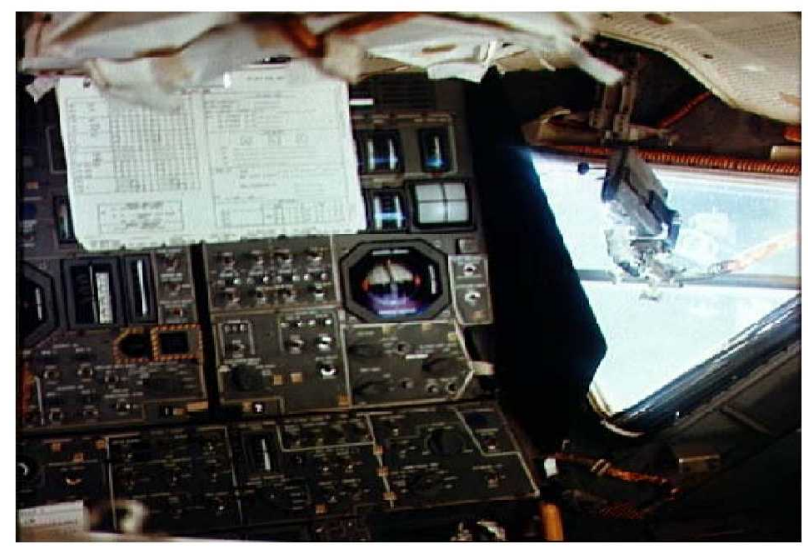

Figure 1. Apollo Lunar Module

The importance of pilot visibility was not only emphasized by trajectory design and window definition, but also, by conducting the Apollo landing task only at specific times and locations to provide optimal sun light on the landing site [5]. The mission was designed around lighting conditions (typically lasting about a week) that would create shadows providing optimal depth perception.

In response to a 2004 Presidential directive to "implement a sustained and affordable human and robotic program to explore the solar system and beyond" [7], NASA initiated the Constellation Program. NASA's Constellation Program is developing a new space transportation system to travel beyond low Earth orbit to initially establish a sustained human presence on the moon and then go to Mars and other destinations. Constellation is developing the Orion Spacecraft, the Altair Lunar Lander, and the Ares I and $\mathrm{V}$ rockets to take humans to the moon to establish a lunar outpost.

Preliminary concepts and operations [8-9] anticipated for the Altair Lunar Lander are contradicting Apollo's experience in the use of a pilot's natural vision as the primary landing aid. First, preliminary design concepts for a Lunar Lander show significantly less external visibility for the astronauts than that provided to the Apollo astronauts. The Altair design will be significantly larger than the Apollo LM and the size of the windows and their location may be severely constrained and non-optimal for pilot visibility. Second, fuel-optimized trajectories do not include a "pitch-up" maneuver, as Apollo did, for pilot visibility through windows. If the need for natural visibility could be eliminated or reduced, significant fuel savings could be gained. Finally, future mission requirements call for frequent landings in the Lunar polar regions for maximum scientific benefit. The polar regions exhibit less than ideally lighted lunar surface locations. The very low sun-angles will create shadows that can mask landing hazards to a pilot's natural vision, such as craters and boulders. Each of these factors emphasizes the importance of providing technological capabilities for unfettered moon surface landings, without sole reliance on a pilot's natural vision.

Based on research from the aviation domain, SV and EV technologies hold significant promise for planetary missions because the technology presents a computer-generated (SV) and real-time (EV) view of the terrain, topography and other significant characteristics (e.g., habitat modules, power generators, etc.) independent of the outside visibility conditions (i.e., darkness, shadow illusions or obscuration, engine effluent/dust), window locations, or vehicle attributes. SV allows unconstrained control of the computer-generated scene lighting, terrain coloring and virtual camera angles which may provide invaluable visual cues to pilots/astronauts, not available from other vision technologies. EV sensors, such as FLIR and LIDAR, provide real-time imagery of surface topography and hazards and can provide real-time integrity monitoring of the $\mathrm{SV}$ database. Vehicle state information (e.g., forward and down velocities, altitude, and fuel remaining) may be conformally overlaid on the SV/EV imagery to enhance trajectory control and overall crew situation awareness especially when compared to the information presentation and data display used for the Apollo LM. 


\section{Current Study}

The objective of this study was to gain a firstorder assessment of whether the benefits of EV/SV technologies, shown in the aviation domain, do indeed carry over to the space domain. This experiment examined the effects of having terrain imagery on space vehicle flight displays during lunar approaches and landings for nominal and off-nominal situations.

The terrain imagery concepts were: (a) EV-only, (b) SV-only, (c) EV and SV, and (d) Baseline (i.e., no SV or EV) Head-Down Display Concepts. These terrain imagery concepts were shown on head-down displays providing a head-up display (HUD)-like format, primary flight reference information display, navigation display (ND), and an ego-centric situation awareness (SA) information display.

The specific research objectives included: (a) evaluating the contribution of $\mathrm{EV}$ and/or SV visualization technologies on vehicle flight displays for approaches and landings to the lunar surface; (b) evaluating the effects of various vehicle external fieldof-views and $\mathrm{EV}$ and/or SV visualization technologies; (c) evaluating SA and workload during terrainchallenged, fuel-optimized approaches; and (d) evaluating the contribution of terrain and hazard display depiction on landing redesignation functionality.

\section{Method}

\section{Subjects}

Eight pilots participated as subjects in this experiment. Because of the powered-lift nature of the Lunar Lander vehicle and the vertical descent and landing task, rotorcraft piloting experience was felt to be important to reduce training time for the simulation. Pilot subject recruitment was conducted, using established NASA protocol, requiring subjects to have a commercial pilot's license, and if possible, rotorcraft experience. Six of the evaluation pilots (EPs) had previous experience flying rotorcraft with an average of 687 hours of flight time.

\section{Simulation Facility}

The experiment was conducted in the Visual Imaging Simulator for Transport Aircraft Systems (VISTAS) III rapid-prototyping workstation at NASA Langley Research Center. VISTAS III is a non-motion platform, nominally used for crew-vehicle interface concept development for transport aircraft (Figure 2).

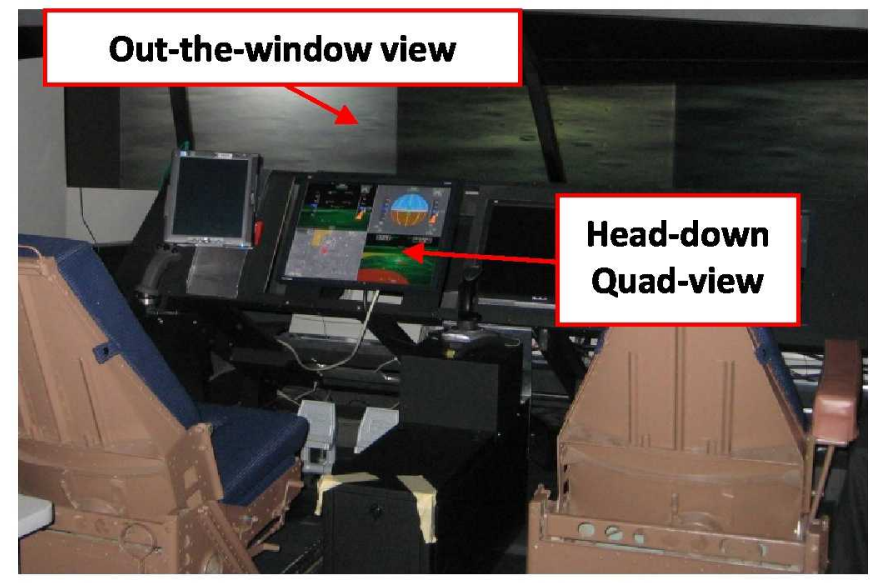

Figure 2. VISTAS III Simulator

For this single pilot experiment, a Logitech Freedom joystick was added for the pilot interface to the lunar lander simulation and had pitch/roll/yaw attitude control, thrust control and 10 programmable buttons [10]. The head-down display concept evaluations were shown on a $1600 \times 1200$ liquid crystal display flat panel with a 22 inch total display diagonal. Four equal-sized display areas ( 9 inches wide by 7 inches high) provided four flight instrument formats: HUD-like information, Primary Flight Display (PFD) information, ND information, and SA information.

The out-the-window (OTW) view consisted of three $1024 \times 1280$ displays with a total resolution of 3072 pixels horizontal by 1280 pixels vertical. The total field-of-view was $72^{\circ}$ horizontal by $30^{\circ}$ vertical with a resolution of 43 pixels/degree. The out the window displays were centered vertically to the pilot design eye point giving an equal $15^{\circ}$ up and down look angle. Note that this look-down angle of $15^{\circ}$ is a limitation of the VISTAS III facility as it doesn't allow the $70^{\circ}$ look-down angle of the Apollo windows.

\section{Vehicle and Lunar Properties}

Available public-release data on Lunar Lander concepts were used to derive mass and inertial properties, from Lunar Orbit Insertion, to the appropriate trajectory initialization point for each run, including nominal Reaction Control System fuel utilization, to that point. The Moon was simulated as a homogeneous, spherical, rotating mass, for purposes of this experiment. A fourth order Runge-Kutta 
integrator was used for state propagation, with a fixed step size of 0.01 seconds.

\section{Lunar Database}

The Apollo 15 landing site was utilized in this experiment because of the availability of higher resolution data and the interesting terrain features it provided. The visual lunar database was created using lunar topology and imagery data collected by the Clementine project. Clementine was launched on January 25, 1994 and collected lunar surface data during the months of February, March, and April of that year. A spherical lunar database was created that covered the entire lunar surface and was built with three topology and imagery resolutions. Database coverage for the entire moon was created using a resolution of approximately 4 kilometers per elevation post, draped with 1 kilometer per pixel imagery. Medium resolution data was used around the area of the Apollo 15 landing site, with approximately 500 meters per elevation post, draped with 125 meter per pixel imagery. The highest resolution data was used in the area immediately surrounding the Apollo 15 landing site. This resolution was 15 meters per elevation post draped with 4 meter per pixel imagery.

The same terrain data were used for the SV, FLIR, and OTW presentations; however, their display presentations were altered to roughly approximate the different sensors and concepts. For the FLIR presentation, the imagery color was converted to green based on the intensity of the true color and the brightness was attenuated based on pixel distance and elevation/azimuth angle. Random noise was also inserted. The SV and OTW presentations were identical except for the addition of boulder hazards in the OTW scene. Fifty boulders were pseudo-randomly placed around the Apollo 15 landing site in a 0.015 degree by 0.015 degree area (Figure 3 ). The boulders ranged from 0.5 meters in diameter to 10 meters in diameter. The same boulders were also visible in the FLIR presentation.

A surface feature (hereinafter referred to as the lunar landing cue) was placed at the landing site so the EP's would have a visual reference to the desired touchdown point (see Figure 3). The lunar landing cue was a circular ring that was 60 meters in diameter, 10 meters thick, and two meters high. The ring was textured with a pseudo lunar surface image and blended into the lunar surface topology. The lunar landing cue was displayed in all three terrain presentations (SV, EV FLIR, and OTW).

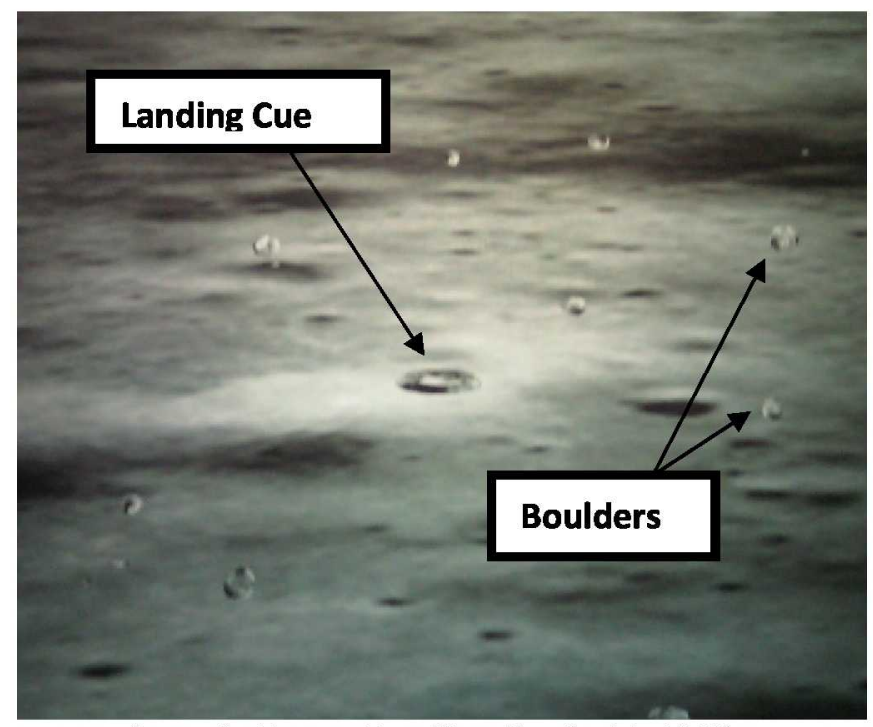

Figure 3. Lunar Landing Cue in OTW View

\section{SV Database}

As mentioned in the lunar database section, the SV database presentation of lunar terrain was identical to that provided OTW except it did not include the boulder hazards. The boulders were not present in the $\mathrm{SV}$ terrain to simulate objects which might be too small to be detected by a terrain data gathering sensor. Further, as the SV terrain is computer generated, actual sun angles do not affect the visibility of the terrain.

\section{EV Sensors}

Two rudimentary, non-physics-based sensors were simulated in this experiment: FLIR and LIDAR. The FLIR presentation was identical to the OTW view of the lunar terrain, including the boulder hazards and the lunar landing cue. It was depicted in green on the display concepts using EV sensor information (see EV and Fused EV/SV display concepts in Figure 4). The LIDAR sensor provided a "flash shot" of the lunar terrain at a $1000 \mathrm{~m}$ altitude on approach. The LIDAR data was not shown directly to the EP, but instead, a simulated "processed" image of computed hazards was shown. "LIDAR-detected" hazards were depicted as transparent, red colored circles (Figure 4) on the display concepts that used EV sensor information. These LIDAR-detected hazards were only displayed after the vehicle had passed through $1000 \mathrm{~m}$ altitude. 


\section{Independent Variable - Display Concepts}

Four flight display concepts (Figure 4) were evaluated by the EPs while performing lunar approaches and landings. Each flight display concept

\section{Baseline Display Concept}

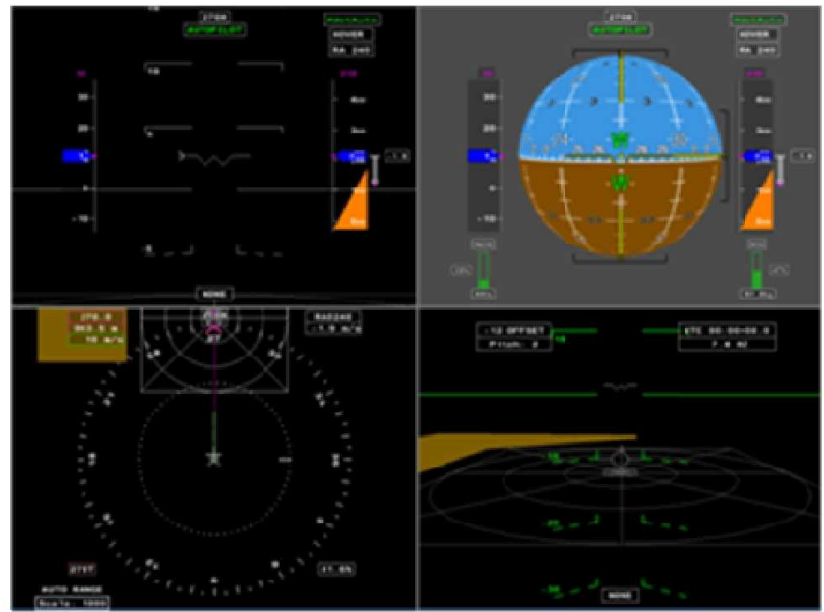

SV Display Concept

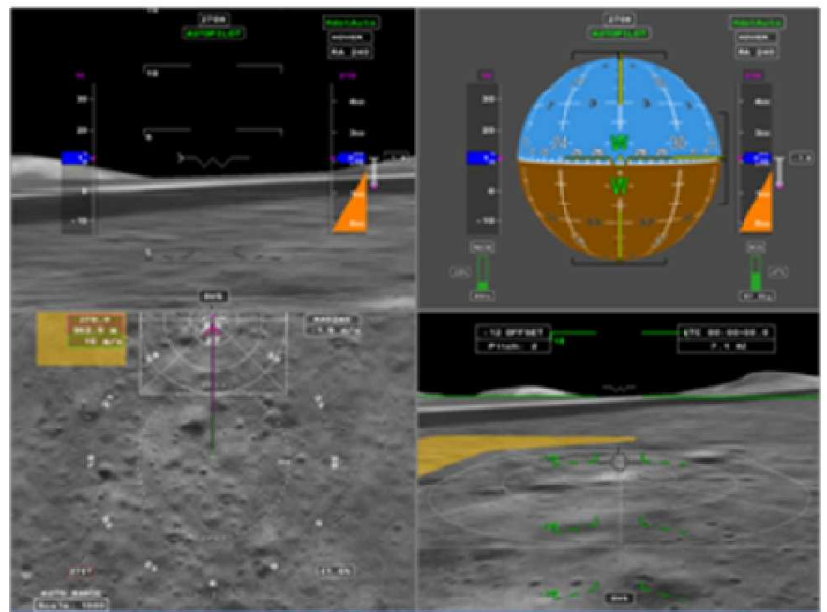

was part of the quad-view of head-down flight instruments. The quad-view included a HUD (upper left window), PFD (upper right window), ND (lower left window), and egocentric Situation Awareness (SA) Display (lower right window).

\section{EV Display Concept}

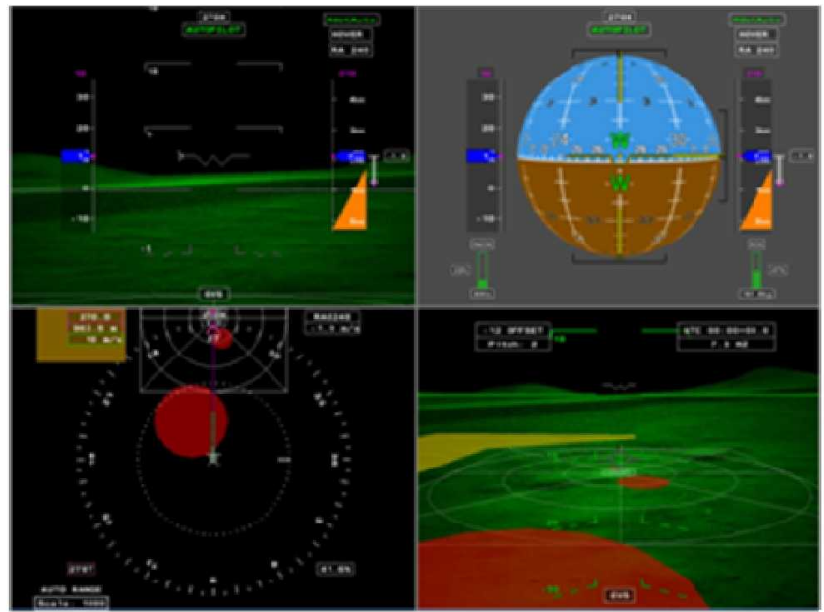

\section{Fused EV/SV Display Concept}

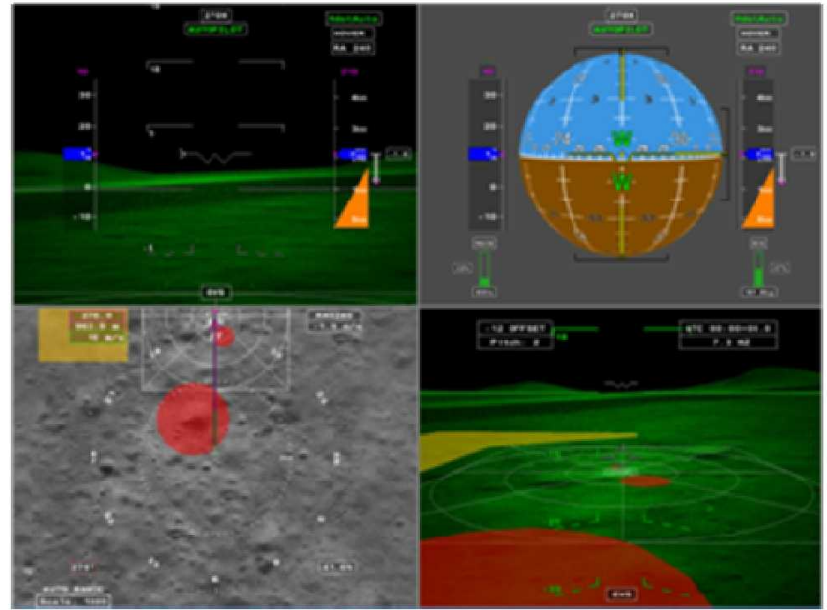

Figure 4. Display Conditions - Baseline (upper left), EV (upper right), SV (lower left), and Fused EV/SV (lower right).

The HUD display was attitude-referenced and showed conformal EV/SV information (when utilized) and symbology.

The PFD was a standard three-axis "eight-ball" providing head-down primary flight reference information. EV and SV information was not added to this display to ensure uncluttered guidance and uncompromised full spatial reference information necessary for the attitude extremes of planetary descent.
The SA display provided ego-centric symbology and EV/SV information (when utilized) with a pilotcontrollable vertical pan of the SV information or simulated EV camera. Pilot-selectable discrete settings for this vertical pan included: Flight Path Marker (FPM)-centering, Nadir-centering, and Waterlinecentering. Slewable up/down viewing control to $+/-90$ degrees was also provided. By using the SA display and its variable viewpoint references, critical terrain and obstacle awareness could be provided, potentially 
without the loss of spatial awareness or critical guidance information.

The ND was a simple two-dimensional, top down view with own-ship position located at the center of the display. The synthetic lunar terrain (when utilized) was layered under the symbology. A touchdown zone was drawn centered on the designated touchdown site.

Detailed descriptions of the flight instruments (HUD, PFD, ND, and SA display) and the symbology used within them can be found in Reference 11.

The symbology used within the four flight instruments of the pilot's quad-view were held constant across each display concept evaluated. Regardless of the display concept, the "no-plume" zones (ambercolored areas within which the vehicle shouldn't fly) and the touchdown zone symbol were always depicted on the ND and SA displays.

Within these four instruments, the type of terrain imagery (EV, SV, fused $\mathrm{EV} / \mathrm{SV}$, or none) was experimentally varied as illustrated in Figure 4.

In the upper left quadrant of Figure 4, the Baseline Concept is shown. This concept did not include any head-down terrain imagery. The EP had to rely on the OTW view and altimeter readings to ascertain the vehicle's height above the lunar terrain.

In the upper right quadrant of Figure 4, the EV Concept is illustrated. The terrain imagery was created from the simulated FLIR and LIDAR-detected hazard information. Boresighting errors, sensor abnormalities and artifacts, vehicle-induced obstructions, or cameraslewing dynamics were not simulated.

In the lower left quadrant of Figure 4, the SV Concept is illustrated. Terrain imagery came from an on-board lunar database, using simulated on-board navigation state information. Errors in the navigation solution would degrade the accuracy of SV information and could cause a notable discrepancy between it and the OTW view.

In the lower right quadrant of Figure 4, the Fused EV/SV Concept is illustrated where the terrain imagery was a fusion of EV and SV information. A simple blending of the EV and SV information was selected for the fusion. Above $335 \mathrm{~m}$ altitude, the terrain imagery was purely SV on the HUD, ND, and SA display. Below $335 \mathrm{~m}$ altitude, the terrain imagery was FLIR on the HUD and SA display and SV on the ND. Simulated LIDAR-detected hazards (red circles within which the vehicle should avoid landing) were added to the ND and SA displays.

\section{Independent Variable - Out the Window Vertical Field-of-Regard}

Two OTW vertical field-of-regard (FOR) views were varied to experimentally test the criticality of OTW vision for the approach and landing task. The two OTW FORs are hereinafter referred to as the Small OTW and Large OTW views. A fuel-optimized approach/landing trajectory (i.e., fast and low, or shallow perspective, with the velocity being arrested just before touchdown) to the lunar surface was used to generate the Small OTW view condition. This type of trajectory is being considered for the Altair lunar missions [8]. An Apollo-like approach/landing trajectory was used to generate the Large OTW view condition. During the final approach phase, Apollo astronauts were required to use natural vision (through the LM windows) to monitor safety of flight and perform landing site assessment.

In VISTAS III, the total OTW view was fixed at $30 \mathrm{deg}$ but simulated body-axis gimbaling (i.e., the crew-occupied body could be rotated independent of the engine housing) was employed to modify the lookdown angle and increase the amount of time and volume of viewable OTW terrain for the EP. For the Small OTW view condition, gimbaling was not used. The crew-occupied body was fixed to the engine housing and the FOR was $+/-15$ degrees from the vehicle boresight. For the Large OTW view condition, gimbaling biased the look-down angle up to 35 degrees look down from the boresight. Gimbaling is not necessarily a desirable or recommended method of providing crew visibility of the landing site, but for this experiment it was used as an expedient method of changing the look-down without changing the task or the guidance and control laws.

\section{Evaluation Tasks}

During an experimental run, EPs performed either an Approach Task or a Landing Task.

\section{Approach Task}

The trajectory for the Approach Task started at approximately $170 \mathrm{~km}$ from the desired landing site, $782 \mathrm{~m} / \mathrm{s}$ groundspeed, and $11400 \mathrm{~m}$ Height Above Touchdown (HAT). A guidance algorithm commanded a geometric fifth order profile in altitude, to a specified endpoint over the mountains $24 \mathrm{~km}$ prior 
to the desired landing site. The commanded longitudinal profile approximated a constant "jerk" (i.e., first derivative of acceleration) profile to a specified control point, located well after the end of this guidance phase. Simulated body axis gimbaling was employed to increase the look-down angle (35 degrees maximum gimbal angle) in the Large OTW view condition.

Pitch attitude started at approximately 60 degrees, and gradually decreased to about $\mathbf{3 0}$ degrees at the end of the run. Gimbaling, provided for the Large OTW view runs only, was linear with range, starting at zero degrees at $45 \mathrm{~km}$ range from the landing site, and reaching the maximum of 35 degrees at $30 \mathrm{~km}$. In the Large OTW view condition, terrain ahead of the vehicle was visible out the window until just prior to the end of run for the nominal cases and for about 15 seconds prior to terrain impact for the guidance failure cases (impact could be avoided in these cases by selecting Abort Mode). For the Small OTW view condition, the terrain in front of the vehicle was not visible in the window for any portion of the nominal or guidance error runs.

The EP's task was to monitor the vehicle state as the autopilot was flying the final approach trajectory, verifying terrain clearance to the mountain range as the landing site was approached. Each of the displays provided key elements in assisting the EP to complete these tasks. When provided, the velocity vector and terrain imagery on the SA Display, as well as OTW terrain cues, were used to assess projected terrain clearance. The PFD information enabled verification that the vehicle autopilot was flying commanded guidance, and provided speed, altitude, sink rate, fuel, and attitude awareness. The HUD provided attitude awareness, and the ND provided position and navigation state awareness.

\section{Landing Task}

The trajectory for the Landing Task varied, depending on the selected OTW view condition. For Small OTW view landings, scenarios started at 14500 $\mathrm{m}$ range, $4100 \mathrm{~m} \mathrm{HAT}$, and $215 \mathrm{~m} / \mathrm{s}$ groundspeed. Large OTW view landings started at $1000 \mathrm{~m}$ range, $200 \mathrm{~m} \mathrm{HAT}$, and $10 \mathrm{~m} / \mathrm{s}$ groundspeed. Initial conditions were chosen to provide approximately five minute run times for each scenario.

For the initial portion of the landing scenarios, the guidance algorithm commanded a geometric fifth order profile in altitude, to a specified control point near the landing site, and constant "jerk" for the longitudinal profile. In the final portion of the landing scenarios, guidance commanded a constant flight path angle to a hover point $45 \mathrm{~m}$ overhead the landing site, with a constant "jerk" profile in groundspeed.

For the Large OTW view condition, pitch attitude started at about two degrees, decreasing gradually to zero over the landing site. For these runs, gimbaling was used, starting at about 12 degrees at the start of run (about $1000 \mathrm{~m}$ range to landing site), and decreasing to zero by about $280 \mathrm{~m}$ range (at which point, the landing site was not visible out the window). The combination of trajectory, pitch attitude, and gimbaling provided about 70 seconds visibility of the landing site out the window, for the Large OTW view runs. In addition, lunar terrain approximately $100 \mathrm{~m}$ downrange of the landing site was visible for approximately 100 seconds in the Large OTW view condition prior to reaching the hover point.

For the Small OTW view condition, pitch attitude started at approximately 55 degrees at the beginning of the run, and gradually decreased to zero over the landing site. The landing site was not visible out the window at any time during these runs. However, lunar terrain approximately $100 \mathrm{~m}$ downrange of the landing site was visible for approximately 31 seconds in the Small OTW view condition. This OTW visibility provided the pilot with some awareness of the terrain height and ground velocities and position, but a natural vision view of the landing site was never available in this condition.

The EP's task was to continue to monitor the autopilot during the landing until $50 \mathrm{~m}$ altitude. At this point, the EP was required to take over control from the autopilot and manually fly the vehicle to the designated landing site. Each of the displays provided key elements in assisting the EP to complete these tasks. When provided, the terrain imagery and velocity vector on the SA Display, as well as OTW terrain cues, were used to assess projected path to the landing site. In the final descent from the hover point to contact, the Nadir View could be used on the SA display to affect fine control of the projected touchdown position, and increase landing accuracy. The PFD enabled verification that the vehicle autopilot was flying commanded guidance, and provided speed, altitude, sink rate, fuel, and attitude awareness. The HUD provided attitude awareness, and the ND provided position awareness. Aural cues were provided as 
additional altitude and sink rate cues. Aural cues consisted of a pre-recorded (i.e., not synthesized) female voice, annunciating the altitude at the following crossing points in meters: $100,50,40,30,20,10,5,4$, 3,2 , and 1 . In addition, the word "contact" was spoken at simulated ground sensor contact.

\section{Non-Normal Runs}

In addition to the nominal final approach and landing maneuvers, the EPs were exposed to 3 unannounced system failures for both OTW views (Small, Large): 1) guidance error on approach, 2) guidance error on landing, and 3) navigation error on landing.

\section{Guidance Error on Approach}

In the approach guidance error scenarios, the flight director guided the vehicle on a trajectory into rising terrain prior to the landing site. This error was simulated by modifying the nominal guidance to use an erroneous altitude ( $465 \mathrm{~m}$ lower than nominal) and lateral position (7000 $\mathrm{m}$ left of path) for the approach phase. With this error inserted, if undetected, the vehicle would impact terrain.

\section{Guidance Error on Landing}

In the landing guidance error scenario, the flight director guided the vehicle to a touchdown point located in a simulated LIDAR-detected hazardous area. Guided trajectories were modified from those used in nominal runs, in that a moderate deviation in along track ( $136 \mathrm{~m}$ beyond landing site) and cross track (61 m right of landing site) position was inserted. If undetected, the guidance would direct the vehicle into a simulated hazardous landing location.

\section{Navigation Error on Landing}

The navigation failure scenario introduced an error in the spacecraft's navigation solution. The landing phase navigation error resulted in the trajectory being $136 \mathrm{~m}$ short (along track deviation) and $61 \mathrm{~m}$ left (cross track deviation) from the nominal landing site. These deviations, if undetected, would drive the vehicle into a simulated hazardous landing location.

\section{Pilot Procedures}

The EPs were instructed to complete the approach or landing if they determined that the maneuver could be completed safely. They were instructed to closely monitor the autoland approach and to land the Lunar Lander vehicle as close as possible to the center of the landing site. If they felt that the final approach maneuver was unsafe, they were instructed to perform an abort. (An abort was initiated by a button press on the joystick.) The EPs were not given the option to take-over manual control of the vehicle until reaching the hover point. It was stressed that initiating an abort should not be taken lightly. However, it was also stressed that if they felt the mission was unsafe for the crew or vehicle that an abort should be performed.

If the EPs felt that the landing site was not safe for landing, they could choose a new landing site based on the OTW view and display concept provided to them. Choosing the new landing site was accomplished through a 4-step redesignation process: 1) press and hold front trigger button on controller; 2) move controller to desired new landing site; 3 ) release trigger button; and 4) press button on side of controller to confirm new landing site.

If an abort was initiated, the evaluation run was ended once terrain clearance was increasing.

The EPs were trained to recognize and understand the terrain imagery provided to them OTW, synthetically through a database, or imaged by a FLIR or LIDAR sensor. They were also trained on crosschecking different symbology elements to verify the integrity of their vehicles' navigation solution. The EPs were trained on both the redesignation and abort procedures. The non-normal runs were neither briefed nor included in the training runs.

Prior to run commencement, the EP was briefed on the type of task (approach or landing) to be completed, the display concept to be evaluated (Baseline, EV, SV, Fused EV/SV), and the OTW view to be used (Small, Large).

\section{Experiment Matrix}

\section{Nominal Run Matrix}

Nominally, eight training runs and twenty experimental runs were completed by each EP. Of the 20 experimental runs, 6 non-normal runs were included to investigate the pilot's awareness and reaction to unexpected events and conditions (e.g., failures). The non-normal data are critical determinants in the underlying safety of spacecraft operations.

For approach runs, the experiment matrix consisted of a full factorial combination of display concept (Baseline, EV, SV) and OTW view (Small, Large) for a total of 6 runs. At the altitudes being 
flown for the approach trajectories, the SV and Fused EV/SV concepts were identical so only one of the two, the SV concept, was chosen for evaluation.

For landing runs, the experiment matrix consisted of a full-factorial combination of display concept (Baseline, EV, SV, Fused EV/SV) and OTW view (Small, Large) for a total of 8 runs.

A within-subjects design was employed for the nominal approach and landing runs.

\section{Off-Nominal Run Matrix}

Each EP flew 6 non-normal runs (3 off-nominal conditions by 2 OTW view conditions): (a) guidance error on final approach, (b) guidance error during landing, and (c) navigation error during landing combined with either (a) a Small OTW view condition or (b) a Large OTW view condition.

A between-subjects design was employed for the non-normal approach and landing runs.

\section{Measures}

During each run, pilot inputs (e.g., landing site redesignation or abort initiation) and landing variables (distance from landing site center, lateral landing speed, and vertical landing speed) were measured for analysis.

After each run, EPs completed a run questionnaire consisting of the NASA Task Load Index (TLX) workload rating [12], Situation Awareness Rating Technique (SART) [12], and Likert-type (5-point) questions specific to monitoring an approach or manually taking over and landing the vehicle. The approach questionnaire (Figure 5) had the EPs self-rate their awareness of lunar surface terrain and terrain hazards as well as their awareness of the vehicle's energy state, attitude, altitude above the lunar surface, and trajectory. Similarly, the landing questionnaire (Figure 6) asked the EPs to self-rate their ability to recognize the landing zone, pick up visual references to aid landing, and complete the landing as well as their awareness of lunar hazards (e.g., boulders, no-fly areas), altitude above the lunar surface, and vehicle attitude.

After data collection was completed, EPs were administered two paired comparison tests: the Situation Awareness - Subjective Workload Dominance (SASWORD) [13] and Subjective Workload Dominance (SWORD) [12] techniques.

\begin{tabular}{|l|c|c|c|c|c|}
\hline $\begin{array}{l}\text { Post-Run APPROACH } \\
\text { Ratings }\end{array}$ & Excellent & Good & Acceptable & Poor & $\begin{array}{c}\text { Absolutely } \\
\text { Unacceptable }\end{array}$ \\
\hline Please rate your: & 1 & 2 & 3 & 4 & 5 \\
\hline $\begin{array}{l}\text { Q1. Awareness of } \\
\text { spacecraft's energy state }\end{array}$ & & & & & \\
\hline $\begin{array}{l}\text { Q2. Awareness of } \\
\text { spacecraft's attitude }\end{array}$ & & & & & \\
\hline $\begin{array}{l}\text { Q3. Awareness of altitude } \\
\text { above the lunar surface }\end{array}$ & & & & & \\
\hline $\begin{array}{l}\text { Q4. Awareness of } \\
\text { spacecraft's trajectory }\end{array}$ & & & & & \\
\hline $\begin{array}{l}\text { Q5. Awareness of lunar } \\
\text { surface terrain and terrain } \\
\text { hazards }\end{array}$ & & & & & \\
\hline
\end{tabular}

Figure 5. Approach Post-Run Questionnaire

\begin{tabular}{|c|c|c|c|c|c|}
\hline $\begin{array}{l}\text { Post-Run LANDING } \\
\text { Ratings } \\
\text { Please rate your: }\end{array}$ & $\begin{array}{c}\text { Excellent } \\
1 \\
\end{array}$ & $\begin{array}{c}\text { Good } \\
2 \\
\end{array}$ & \begin{tabular}{|c} 
Acceptable \\
3 \\
\end{tabular} & $\begin{array}{c}\text { Poor } \\
4 \\
\end{array}$ & \begin{tabular}{|c|} 
Absolutely \\
Unacceptable \\
5 \\
\end{tabular} \\
\hline $\begin{array}{l}\text { Q1. Ability to recognize } \\
\text { the designated landing } \\
\text { zone }\end{array}$ & & & & & \\
\hline $\begin{array}{l}\text { Q2. Awareness of altitude } \\
\text { above the lunar surface }\end{array}$ & & & & & \\
\hline $\begin{array}{l}\text { Q3. Awareness of } \\
\text { spacecraft's attitude }\end{array}$ & & & & & \\
\hline $\begin{array}{l}\text { Q4. Ability to pick up } \\
\text { visual references to aid } \\
\text { landing }\end{array}$ & & & & & \\
\hline $\begin{array}{l}\text { Q5. Ability to complete the } \\
\text { landing }\end{array}$ & & & & & \\
\hline $\begin{array}{l}\text { Q6. Awareness of lunar } \\
\text { hazards (e.g, boulders, } \\
\text { no fly areas, etc.) }\end{array}$ & & & & & \\
\hline
\end{tabular}

Figure 6. Landing Post-Run Questionnaire

\section{Test Conduct}

The EPs were given a 1-hour briefing to explain the experiment purpose, display concepts, pilot procedures, and the evaluation tasks. After the briefing, a 1-hour training session in VISTAS-III was conducted to familiarize the EPs with the vehicle's handling qualities, display symbologies, pilot procedures, and controls. The EP's responsibility for maintaining safe operations at all times was stressed. Data collection lasted approximately 4.5 hours and was followed by debriefings which included the SWORD/SA-SWORD paired comparisons tests. The entire session including lunch and breaks lasted approximately 8 hours.

\section{Results}

Nominal and off-nominal approach and landing run results are presented.

The nominal run results are divided into 4 sections: 1) landing performance, 2) mental workload, 3) situation awareness; and 4) post-run questionnaire ratings. The mental workload section includes EP post-run NASA TLX ratings and post-test SWORD 
ratings for the approach and landing tasks. The Situation Awareness section includes EP post-run SART ratings and post-test SA-SWORD ratings.

The off-nominal results are divided into 3 sections: 1) guidance error on approach, 2) guidance error on landing, and 3) navigation error on landing. Within the off-nominal results sections, EP reactions to these errors are discussed. In addition, the EPs postrun SART ratings, NASA TLX ratings, and post-run questionnaire ratings are presented in each section.

For the landing performance measures and postrun SART, TLX, and questionnaire ratings, analysis of variance (ANOVA) techniques were used with display concept and OTW view (Small, Large) as the main factors. For the post-test SWORD and SA-SWORD paired comparisons, simple ANOVAs were conducted across the display concept only. When necessary, Student-Newman-Keuls (SNK) post-hoc tests with $\alpha$ set at 0.05 were performed.

\section{Landing Performance}

Landing performance was assessed for the nominal runs using distance from landing site center (nominal value of $0 \mathrm{~m}$ ), lateral landing speed (nominal value of $0 \mathrm{~m} / \mathrm{s}$ ), and vertical landing speed (nominal value of $-1 \mathrm{~m} / \mathrm{s}$ ).

\section{Distance from Landing Site Center}

An ANOVA revealed no significant differences ( $>0.05$ ) for display concept (Baseline, EV, SV, Fused EV/SV), OTW view (Small, Large), or the interaction between these two factors for the distance from landing site center measure. The overall mean distance from the landing site center was $0.23 \mathrm{~m}$, with a standard deviation, $\sigma=0.22 \mathrm{~m}$.

\section{Landing Speeds}

Similar to distance from the landing site results, a multivariate ANOVA (MANOVA) revealed that none of the main factors or their interactions were significant $(p>0.05)$ for either the lateral or vertical landing speeds, with an overall mean lateral landing speed of $0.002 \mathrm{~m} / \mathrm{s}$ (with $\sigma=0.008 \mathrm{~m} / \mathrm{s}$ ) and vertical landing speed of $-0.04 \mathrm{~m} / \mathrm{s}$ (with $\sigma=0.08 \mathrm{~m} / \mathrm{s}$ ).

\section{Mental Workload}

Mental workload was assessed after each experimental run, using the NASA TLX, and post-test, using the SWORD technique.
NASA TLX is a multi-dimensional rating procedure that derives an overall workload score based on a weighted average of ratings on six subscales (mental demand, physical demand, temporal demand, performance, effort, and frustration level). The NASA TLX subscales went from 0 (Low) to 100 (High) for the workload ratings and the raw weighted scoring procedure was used. TLX ratings were provided for every data run and experimental condition.

EPs were administered the paired-comparison SWORD scale that enabled comparative ratings of mental workload. Mental workload was defined for the EPs as "the amount of cognitive resources available to perform a task and the difficulty of that task." The paired-comparison test was structured to only compare the display concepts (i.e., Baseline, EV, SV, Fused EV/SV) flown by the EP. It did not include the experimental variation of Small and Large OTW views.

\section{NASA TLX for Nominal Runs}

Approach Operations: An ANOVA revealed that display concept (Baseline, EV, SV), OTW view (Small, Large), and the second order interaction between these two factors were not significant $(p>0.05)$ for mental workload ratings during the Approach task. The overall mean approach workload rating was 26 , indicating a moderately-low mental workload level during the lunar landing runs.

Landing Operations: An ANOVA revealed that display concept (Baseline, EV, SV, Fused EV/SV), OTW view (Small, Large), and the interaction between these two factors was not significant $(p>0.05)$ for pilot mental workload ratings during landing operations. The overall mean landing workload rating was 40 , indicating a little less than moderate mental workload level during the lunar landing runs.

\section{SWORD}

The post-test paired-comparison SWORD data indicated that display concept $(\mathrm{F}(3,28)=138.34$, $\mathrm{p}<0.001$ was highly significant for the pilot ratings of mental workload. Post-hoc tests (SNK using $\alpha=0.05$ ) showed two unique subsets for the mental workload ratings with the 4 display concepts: 1) Fused EV/SV, $\mathrm{EV}$, and SV display concepts (lowest workload) and 2) Baseline (highest workload).

\section{Workload Discussion}

TLX ratings indicate that overall pilot workload was very manageable and that there were no significant 
display differences or OTW view differences for this measure in either the approach or landing task. As expected, EPs rated the monitoring (approach) task as requiring less mental workload than the flying (landing) task.

Post-test SWORD ratings revealed that mental workload was significantly reduced when terrain imagery was provided on the EV, SV, and Fused EV/SV displays compared to when it wasn't (with the Baseline concept). The SWORD mental workload ratings did not consider the OTW vertical view provided to the EP.

\section{Situation Awareness}

Situation awareness was assessed after each experimental run, using the post-run SART, and posttest, using the SA-SWORD measures.

SART is a multi-dimensional rating technique using the constructs of: 1) demand on attentional resources; 2) supply of attentional resources; and, 3) understanding. From these components, the SART rating is "understanding" reduced by the difference of "demand" minus "supply" (i.e., SART = \{(understanding) - (demand - supply)\}). SART ratings were provided for every data run and configuration.

Similar to the SWORD described above, the SASWORD is a paired-comparison technique that provides relative situation awareness ratings. For these comparisons, SA was defined as "the pilot's awareness and understanding of all factors that will contribute to the safe flying of their aircraft under normal and nonnormal conditions." The paired-comparison test was structured to compare only the display concepts (i.e., Baseline, EV, SV, Fused EV/SV) flown by the EP. Note that this comparison did not explicitly include the experimental variation of Small and Large OTW views.

The SA-SWORD measure differs from the postrun SART measure construct as it is a pairedcomparison test, it was administered post-test for all configurations, and the underlying definition and construct for the SA ratings are different.

\section{SART for Nominal Runs}

Approach Operations: An ANOVA revealed that display concept (Baseline, EV, SV) was significant $(\mathrm{F}(2,14)=4.97, \mathrm{p}<0.05)$ for post-run approach $\mathrm{SA}$ ratings, but OTW view (Small, Large) and the second order interaction between display concept and OTW view were not significant $(\mathrm{p}>0.05)$ for this measure. Post-hoc tests showed that the Baseline concept provided significantly lower SA than either the EV or SV display concepts. There were no significant differences between the EV and SV display concepts for this SA measure.

Landing Operations: An ANOVA revealed that display concept (Baseline, EV, SV, Fused EV/SV) was significant $(F(3,21)=3.36, p<0.05)$ for post-run landing SA ratings, but OTW view (Small, Large) and the second order interaction between display concept and OTW view were not significant $(p>0.05)$ for this measure. Post-hoc tests showed two overlapping subsets for the display concepts: 1) Baseline, EV, SV concepts (lower SA) and 2) EV, SV, Fused EV/SV concepts (higher SA). The Baseline concept had significantly less SA than the Fused EV/SV concept during nominal landing runs.

\section{SA-SWORD}

The post-test SA-SWORD data indicate that display concept $(F(3,28)=36.16, p<0.001)$ was highly significant for the pilot ratings of SA. Post-hoc tests (SNK using $\alpha=0.05$ ) showed three unique subsets for the SA ratings with the 4 display concepts: 1) Fused EV/SV (highest SA); 2) EV and SV; 3) Baseline (lowest SA).

\section{Situation Awareness Discussion}

Having terrain imagery on the flight displays appears to have a more profound effect on SA ratings than the amount of OTW visibility provided to the pilots for both the landing task and approach task evaluated in this experiment. Statistically significant post-run SART rating differences indicated SA improvements when terrain imagery was used in the display concepts compared to when it wasn't in the Baseline condition.

The absence of SA differences in the landing task for the OTW visibility differences may have been attributable to the fact that a Head-Up Display was not available, so target redesignation required head-down information. Also, in the manual flying portion of the task - from hover to touchdown - the small and large OTW views were the same and neither provided a natural vision view of the landing zone.

Interestingly, for the landing task, EPs only found significant SA improvements when the terrain imagery was with the fused EV/SV concept compared to the 
Baseline. The SV and EV conditions were not significantly different than either of these in the postrun SART measure (but they were better than the Baseline in the post-test SA-SWORD comparisons). This result possibly indicates the EP's need for independent sources of terrain information head-down, for sufficiency and comparative purposes.

Similar to the post-run landing SART results, the post-test SA-SWORD ratings revealed that the best SA was found with the fused EV/SV concept. (These post-test SA-SWORD ratings did not consider the OTW vertical view provided to the EP.)

\section{Post-Run Questionnaire Ratings}

\section{Approach Operations}

A MANOVA on the post-run approach questions (Figure 5) revealed that display concept was significant for Question 4 - awareness of the spacecraft's trajectory $(F(2,14)=6.94, p<0.01)$ and Question 5 awareness of lunar surface terrain and terrain hazards $(F(2,14)=23.72, p<0.01)$ and that OTW view was significant for Question 2 - awareness of spacecraft's attitude $(\mathrm{F}(1,7)=5.65, \mathrm{p}<0.05)$.

Post-hoc tests revealed two unique subsets for the display concepts with regard to awareness of the spacecraft's trajectory: 1) EV (mean=1.8) and SV (mean=2.1) concepts provided "good" awareness, and $2)$ the Baseline concept (mean=2.8) provided "adequate" awareness. Similarly, post-hoc tests revealed two unique subsets for awareness of lunar surface terrain and terrain hazards: 1) EV (mean=2.3) and SV (mean=2.2) concepts provided "good" awareness and 2) the Baseline concept (mean=4.1) provided "poor" awareness.

Both the Small (mean=1.8) and Large (mean=2.0) OTW view conditions were rated as providing "good" awareness of the spacecraft's attitude. Although statistically different, the differences in ratings for these two OTW views are operationally inconsequential.

\section{Landing Operations}

A MANOVA (and subsequent post hoc tests) on the post-run landing questionnaire ratings (Figure 6) revealed that for:

Question 1 - ability to recognize the designated landing zone - both display concept $(\mathrm{F}(3,21)=6.44$, $\mathrm{p}<0.01)$ and OTW view $(\mathrm{F}(1,7)=22.87, \mathrm{p}<0.01)$ were significant. The Baseline concept (mean=2.4) was rated as being significantly different than the $\mathrm{EV}, \mathrm{SV}$, and Fused EV/SV concepts (mean=1.5) but all display concepts were in the "acceptable" to "good" range for this measure. Although statistically different, the Large OTW view (mean=1.5) and the Small OTW view (mean=1.9) ratings were both in the "good" range for this measure.

Question 2 - awareness of altitude above the lunar surface - OTW view $(F(1,7)=22.87, p<0.01)$ was significant. Although statistically different, the Large OTW view (mean=1.4) and the Small OTW view (mean=1.8) ratings were both in the "good" range for this measure.

Question 3 - awareness of spacecraft's attitude neither display concept nor OTW view were significant $(\mathrm{p}>0.05)$.

Question 4 - ability to pick up visual references to aid landing - display concept $(\mathrm{F}(3,21)=6.41, \mathrm{p}<0.01)$ was significant. The Baseline concept (mean $=2.6$ ) was rated as being significantly different than the EV, SV, and Fused EV/SV concepts (mean=1.7) but all display concepts were in the "acceptable" to "good" range for this measure.

Question 5 - ability to complete the landing both display concept $(F(3,21)=6.36, p<0.01)$ and OTW view $(F(1,7)=5.72, p<0.05)$ were significant. The Baseline concept (mean=2.1) was rated as being significantly different than the EV, SV, and Fused EV/SV concepts (mean=1.4) but all display concepts were in the "good" range for this measure. Although statistically different, the Large OTW view (mean=1.4) and the Small OTW view (mean=1.8) ratings were both in the "good" range for this measure.

Question 6 - awareness of lunar hazards (e.g., boulders, no fly areas - both display concept $(F(3,21)=6.32, p<0.05)$ and OTW view $(F(1,7)=8.0$, $\mathrm{p}<0.01)$ were significant. The Baseline concept (mean=2.9) was rated as being significantly different than the EV, SV, and Fused EV/SV concepts (mean=1.8) but all display concepts were in the "acceptable" to "good" range for this measure. Although statistically different, the Large OTW view (mean=1.8) and the Small OTW view (mean=2.3) ratings were both in the "good" range for this measure.

\section{Post-Run Questionnaire Ratings Discussion}

No operationally significant OTW view differences were found in either the approach or landing post-run questionnaire ratings. In general, 
display concepts with EV and/or SV imagery were preferred over the no-terrain Baseline condition as they improved an EP's ability to land safely due to increased awareness of elements such as the landing site, terrain, and hazards within the lunar landing environment.

\section{Off-Nominal Scenarios}

\section{Guidance Error on Approach}

All EPs who experienced a guidance error on approach recognized the potential flight into terrain and aborted the approach. However, one EP while flying with the EV display concept in the Small OTW view condition pressed the abort button too late and actually impacted the lunar terrain. The EP's only sources of terrain information in this run were the EV imagery and the radar altitude information on the displays as there was no terrain visible in the Small OTW view.

EP post-run Approach Questionnaire ratings (Figure 5, Question 5) revealed that the Baseline display concept offered significantly less awareness of lunar surface terrain and terrain hazards, $F(3,8)=6.44$, $\mathrm{p}<0.05$, compared to the other three concepts tested that employed EV and/or SV terrain imagery for the approach guidance error runs. The Baseline concept had a mean rating of 3.8 which equated to "poor awareness" on the post-run Likert scale and the other concepts had a mean rating of 1.5 which equated to "good awareness" on that same scale. There were no significant differences between the Small and Large OTW view conditions for this questionnaire rating measure. In addition, there were no significant differences $(p>0.05)$ between the display concepts or the OTW view conditions for the EP SART ratings, TLX ratings, or remaining four Post-Run Questionnaire ratings (Figure 5) for the guidance error on approach runs.

\section{Guidance Error on Landing}

All but one landing guidance error run was detected by the EPs. This one undetected error run was with the Baseline concept with Small OTW view. For this one run, the EP didn't have the lunar landing cue head-down on the Baseline display concept or visible within the Small OTW view. The only cues were the terrain over $100 \mathrm{~m}$ downrange of the landing site to recognize s/he wasn't landing in the correct location.

Post-run EP ratings (Figure 6, Question 6) for the guidance error on landing runs indicated that EPs had significantly $(F(3,128)=6.0, p<0.05)$ better awareness of lunar hazards (e.g., boulder, no fly areas) with the three concepts employing EV and/or SV than when using the Baseline concept. EPs also reported (Figure 6, Question 1) they were significantly $(F(3,8)=4.21$, $p<0.05$ ) better at recognizing the designated landing zone with the EV and Fused EV/SV concepts compared to the Baseline concept. Post-hoc tests revealed two overlapping subsets for the display concepts: 1) EV (mean rating=1.5), Fused EV/SV (mean rating=2.0), SV (mean rating=2.3) and 2) Fused $\mathrm{EV} / \mathrm{SV}, \mathrm{SV}$, and Baseline (mean rating=3.5) for this measure. There were no significant display concept differences for EP SART ratings, TLX ratings or remaining post-run questionnaire ratings (Figure 6) for the guidance error on landing runs.

Post-run SART and Landing Questionnaire ratings (Figure 6, Question 5) indicated that EPs had significantly higher SA $(F(1,8)=7.35, p<0.05)$ and better ability in completing the landing $(F(1,8)=6.67$, $\mathrm{p}<0.05$ ) when using the Large OTW view compared to the Small OTW view for the landing guidance error runs. The Large OTW view (mean=1.8) was in the "good" range for the EP's ability to complete the landing, but the Small OTW view (mean=3.4) was approaching the "poor" range in the EP's ability to complete the landing. There were no significant OTW view differences for EP TLX ratings or the other postrun landing questionnaire ratings for the guidance error on landing runs.

\section{Navigation Error on Landing}

All EPs who experienced a navigation error with either the SV or Baseline display concepts either failed to recognize the failure or re-designated but landed on another hazard (i.e., a simulated LIDAR-detected hazard). For all EV and Fused EV/SV display runs, EPs recognized the navigation error and were able to re-designate to a safe landing site.

Post-run Landing Questionnaire ratings (Figure 6, Questions 1 and 6) revealed that the EV and Fused EV/SV display concepts were rated as being significantly easier for recognizing the designated landing zone $(F(3,8)=5.08, p<0.05)$ and for awareness of lunar hazards such as boulders and no fly zones $(F(3,8)=6.12, p<0.05)$ than the Baseline concept. The Baseline concept was also rated (Figure 6, Question 4) as being significantly worse in aiding the pilot in picking up visual cues to aid landing $(F(3,8)=5.82$, $\mathrm{p}<0.05$ ) than the EV, SV, and Fused EV/SV display 
concepts. There were no significant display concept differences for EP SART ratings, TLX ratings or remaining post-run questionnaire ratings (Figure 6) for the landing navigation error runs.

There were also no significant $(\mathrm{p}>0.05)$ OTW view differences for the EP SART ratings, TLX ratings, or any of the post-test questionnaire ratings (Figure 6) for the landing navigation error runs.

\section{Off-Nominal Scenario Discussion}

Regardless of the display concept and OTW view condition evaluated, EPs recognized and correctly reacted (by performing an abort) to the approach guidance error runs. However, one abort, while monitoring the approach with the EV display concept in the Small OTW view condition, was initiated too late and the vehicle was unable to gain altitude and impacted the lunar terrain. Even though the EP had been trained in the time needed for the vehicle to retard its downward velocity and begin gaining altitude once an abort had been initiated (and had EV terrain imagery on the flight displays), this result demonstrated a lack of timely terrain awareness. The data, otherwise, suggests that the tested display or OTW visibility conditions were all sufficient to circumvent this off-nominal condition.

EPs correctly identified unannounced guidance errors during landings when using the display concepts with EV, SV or Fused EV/SV display imagery for both OTW view conditions and when using the Baseline concept with the Large OTW view. However, one run (of the two tested landing guidance error runs) was not recognized by the EP while using the Baseline concept with the Small OTW view, most likely due to not being able to cross-check the lunar landing cue in the EV or SV imagery on the headdown flight displays and OTW with natural vision.

All landing navigation error runs were detected and redesignated to a "safe" landing site when using the EV and Fused EV/SV display concepts with either OTW condition. The results showed that the EV imagery provided an intuitive and obvious visual methodology to monitor the validity of the vehicle's navigation solution. However, all EPs who experienced a navigation failure with either the SV or Baseline displays (using either the Small or Large OTW view conditions) either failed to recognize the failure or re-designated but landed in a simulated hazardous area. For the Baseline (no terrain imagery provided) and SV display concepts, the EPs had to rely on the OTW view of the lunar landing cue (only available in the Large OTW view) and recognition of the lunar terrain features (e.g., boulders on the surface) and their position with respect to these cues to alert them of a poor navigation solution.

\section{Concluding Remarks}

An experiment was conducted evaluating the contribution of enhanced vision (EV) and/or synthetic vision (SV) to displays of a Lunar Lander vehicle during both nominal and off nominal approaches and landings to the lunar surface. As expected, manual landing performance was excellent (within a meter of the landing site center) for the nominal runs regardless if terrain imagery was provided or not provided on the Lunar Lander flight displays. Significant SA improvements were found with the Fused EV/SV display concept compared to the Baseline condition that had no terrain imagery during normal approach and landing operations. Mental workload was manageable for all display concepts tested. There were no significant OTW view differences for pilot SA or workload ratings which may indicate that fuel-efficient approaches may be feasible if terrain imagery is provided on the head-down flight displays.

Subjective and objective off-nominal results support the inclusion SV and EV imagery on fight deck displays for lunar approach and landing, matching our expectations based on aviation-domain research with these technologies. These data emphasize the importance of having a real-time method of monitoring the navigation performance. As shown here, real-time sensors, such as EV, are one method to perform this function as a complement to SV.

\section{References}

[1] Bailey, R. E., L. J. Kramer, L. J. Prinzel III, 2007, Fusion of Synthetic and Enhanced Vision for AllWeather Commercial Aviation Operations, In NATO Human Factors and Medicine Symposium on Human Factors and Medical Aspects of Day/Night All Weather Operations: Current Issues and Future Challenges, Tech. Rep. No. NATO RTO-HFM-141, Neuilly-sur-Seine, France, RTO, pp. 11-1-11-18.

[2] Prinzel, L. J., L. J. Kramer, 2006, Synthetic Vision Systems, In W. Karwowski (Ed.), International Encyclopedia of Ergonomics and Human Factors, Philadelphia, PA, Taylor \& Francis, pp. 25-1 - 25-10. 
[3] Prinzel, L., C. Wickens, 2009, Synthetic Vision Systems, In L. Prinzel \& C. Wickens (Eds.), International Journal of Aviation Psychology (Special Issue), 19(1-2), Philadelphia, PA, Taylor \& Francis, pp. 99-104.

[4] Kramer, Lynda J., Steven P. Williams, Randall E. Bailey, 2008, Simulation Evaluation of Synthetic Vision as an Enabling Technology for Equivalent Visual Operations, In Proceedings of SPIE Enhanced and Synthetic Vision Conference 2008, 6957, Orlando, FL, pp. K-1-K-15.

[5] Slayton, D., 1969, Apollo: Past, Present and Future, In 13th Symposium Proceedings of the Society of Experimental Test Pilots, Beverly Hills, CA.

[6] Hackler, C., J. Brickel, H. Smith, D. Cheatham, 1968, Lunar Module Pilot Control Considerations, Tech. Rep. NASA TN D-4131, Washington, DC, NASA.

[7] NASA, 2004, The Vision for Space Exploration, Washington, DC, NASA.

[8] Brady, T., J. Schwartz, 2007, ALHAT System Architecture and Operational Concept, IEEE Aerospace Conference, Big Sky, Montana.

[9] National Research Council, 2007, The Scientific Context for Exploration of the Moon: Final Report, Washington DC, National Academy of Sciences.

[10] Williams, Steven P., Jarvis J. Arthur, Kevin J. Shelton, Lawrence J. Prinzel III, R. Michael Norman, 2008, Synthetic Vision for Lunar and Planetary Landing Vehicles, In Proceedings of SPIE Enhanced and Synthetic Vision Conference 2008, 6957, Orlando, FL, pp.6-1-6-12.

[11] Prinzel III, Lawrence J., Lynda J. Kramer, R. Michael Norman, Jarvis J. Arthur III, Steven P. Williams, Kevin J. Shelton, 2009, Synthetic and Enhanced Vision System for Altair Lunar Lander, In Proceedings of the 13th International Symposium on Aviation Psychology, Dayton, OH, Wright State University.

[12] AIAA, 1993, Guide to Human Performance Measurement, Washington DC, American Institute of Aeronautics and Astronautics.

[13] Vidulich, M. A., E. R. Hughes, 1991, Testing a Subjective Metric of Situation Awareness, In Proceedings of the Human Factors \& Ergonomics
Society, 35th Annual Meeting, Santa Monica, CA, Human Factors Society, pp. 1307-1311.

28th Digital Avionics Systems Conference

October 25-29, 2009 